\title{
Comunicación

\section{Elecciones, medios y publicidad política en América Latina: los claroscuros de su regulación}

\section{FRANCISCO DE JESÚS ACEVES GONZÁLEZ1}

Como una consecuencia inevitable de la creciente mediatización observada en el desarrollo de las contiendas electorales, la mercadotecnia política, específicamente el uso de la publicidad política televisiva, ha terminado por imponerse como el instrumento fundamental al que se ajustan las estrategias de campaña de los partidos políticos en los países democráticos. El propósito del presente trabajo es analizar, desde una perspectiva comparada, la regulación que se ha establecido respecto al carácter mediático de las campañas, particularmente en lo relativo a la publicidad política en las legislaciones electorales en 19 países latinoamericanos en 2006.

PALABRAS CLAVE: legislación electoral, publicidad política, campañas electorales.
As an inevitable consequence of the increasing media promotion observed in the development of the electoral campaigns, political marketing research, specifically the use of the televised political publicity, has ended up prevailing as the fundamental instrument to which the strategies of campaign of the political parties in the democratic countries adjust. The intention of the present work is to analyze from a comparative perspective the regulation that has been established with respect to the promotional characteristics of the campaigns, particularly with respect to political publicity, in elections of 19 Latin American countries that were taking place in 2006.

KEY WORDS: electoral laws, political advertising, electoral campaigns.

1 Universidad de Guadalajara, México

Correo electrónico: fracegon@yahoo.com.mx

El autor expresa su reconocimiento a Elsa Noemí Mayorga Ochoa, cuya contribución en la captura y procesamiento de información resultó fundamental para la conclusión de este trabajo. 


\section{INTRODUCCIÓN}

Como una consecuencia inevitable de la creciente mediatización observada en el desarrollo de las contiendas electorales, la mercadotecnia política, específicamente el uso de la publicidad política televisiva, ha terminado por imponerse como el instrumento fundamental al que se ajustan las estrategias de campaña de los partidos políticos en los países democráticos. Más allá de las modificaciones que la adopción de estas herramientas han provocado en el carácter y desarrollo de las contiendas electorales, su irrupción ha generado un debate acerca de su impacto en los procesos electorales. La discusión abarca diversos tópicos. Por una parte, se mantiene vigente la añeja discusión sobre los posibles efectos de la publicidad mediática y su capacidad de influir en la definición del voto de sus audiencias. Aunque en este punto todavía hay quienes reivindican el paradigma lazarsfeldiano de los "efectos limitados", se han venido imponiendo diversas perspectivas teóricas, como la agenda-setting (Stevenson et al., 2001 y McCombs and Kiousis, 2004), el priming (Iyengar y Kinder, 1993) y el framing (Shah et al., 1996), que ratifican la importancia de los medios en la percepción que las audiencias construyen sobre su entorno. Por otra parte, se insiste que a consecuencia de esta mediatización, el debate político se ha degradado (Sartori, 1992 y 1998). Finalmente, algunos estudiosos han comenzado a plantearse algunas cuestiones relacionadas con la irrupción de la publicidad política mediática: ¿Ha mejorado la calidad comunicacional de los procesos electorales? ¿Ha contribuido a la profundidad del debate político? ¿Ha proporcionado al elector información útil y necesaria para la definición de su voto? Son preguntas cuya preocupación va más allá de la "eficiencia" del instrumento y se centra en sus efectos sobre el desarrollo de la democracia, específicamente sobre la calidad de la misma.

En lo que todos coinciden es en el reconocimiento de la centralidad de los medios masivos de comunicación en la conformación del espacio político (Dahlgren, 1997), y que debido a ello "la configuración de la política actual se encuentra capturada por 'la lógica y la organización de los medios' que la encuadran y estructuran, y que -siguiendo a Castells (1999)- en dicho encuadramiento se encuentra una de "las fuentes fundamentales de la crisis de la democracia en la era de la información”. 
Derivado de este carácter irreversible de la intervención de los medios en los procesos políticos, particularmente en las contiendas electorales, se ha observado una creciente regulación sobre su papel y sus actividades en las legislaciones que los países latinoamericanos han emitido sobre la materia. Sin embargo, los estudios que abordan el tema se encuentran todavía en un estado embrionario (Lauga y García Rodríguez, 2007).

A lo largo del texto se abordará la irrupción de las campañas mediáticas en los procesos electorales y su "americanización"; la oleada democratizadora de la década de 1980 y su efecto en las legislaciones electorales de América Latina; enseguida, se expondrá el procedimiento metodológico sobre el que se sustenta el análisis y se presentarán los hallazgos de la investigación, para concluir con el señalamiento de los claroscuros de la legislación.

\section{IRRUPCIÓN DE LA PUBLICIDAD POLÍTICA}

EN AMÉRICA LATINA

Existe una coincidencia entre los estudiosos del tema en ubicar el nacimiento del marketing político, en la campaña instrumentada por el candidato republicano Dwigth Eisenhower en su camino a la presidencia de Estados Unidos en 1952. Maarek (1997), por su parte, identifica tres etapas en la evolución del marketing político norteamericano. La primera (1952-1960) a la que denomina "infancia" se caracteriza por la sustitución del discurso tradicional de la retórica política por los mensajes breves y directos, así como por el uso intensivo de los medios de comunicación, particularmente los spots radiofónicos y televisivos. La de la "adolescencia" (1960-1976) en la que se depura y profundiza el uso del spot, y la de la "madurez", o estado adulto que la sitúa a partir de los años ochenta, cuando la televisión se convierte en el medio principal de publicidad política, acaparando la mayor parte del financiamiento que los partidos destinan a sus campañas y se observa una creciente incidencia de la publicidad negativa en el desarrollo de las contiendas políticas.

De acuerdo con esta periodización, los primeros balbuceos del marketing político, en América latina, correspondieron al periodo 
de la adolescencia, y se manifestaron, principalmente, en la incorporación de expertos en el manejo de los medios publicitarios en los equipos de campaña, específicamente consultores extranjeros y publicistas locales.

La experiencia latinoamericana en el campo de la mercadotecnia política, según afirmación de Domínguez (1983), arranca en Venezuela en 1968, cuando "los partidos del estatus, AD y COPEI, contratan los servicios de Joe Napolitan y David Garth" como consultores de las campañas de sus respectivos candidatos. Pero es en 1973, con la campaña del candidato de Acción Democrática, Carlos Andrés Pérez, denominada "El hombre que camina" -mediante la conjunción del consultor norteamericano Joe Napolitan y los talentos locales Renny Ottolina y José Enrique "Chelique" Sarabia, este último compositor del tema musical "El caminante", que se convirtió en el himno de la campaña, así como el trabajo de la empresa encuestadora Gallup-, que la publicidad política toma carta de naturalización en los procesos electorales.

En Argentina, justo en el proceso de transición de la dictadura militar a los gobiernos civiles, Muraro (1990) destaca el "uso sistemático de toda la batería de técnicas inherentes al marketing y la publicidad política" en la campaña de Raúl Alfonsin, candidato de la Unión Cívica Radical en las elecciones presidenciales de 1983.

En el caso de Chile, la publicidad política no surgió en una contienda electoral sino en el marco del proceso plebiscitario de 1988, en el que se dirimía la permanencia de Pinochet en el poder, proceso que culminó el 5 de octubre con la victoria de la franja del "No" y el fin de la dictadura. Ante la ausencia de pluralidad en los medios de comunicación y de estrictas restricciones a la libertad de expresión, se constituyó una "franja política" que jugó un papel fundamental en la estrategia comunicacional de los partidos de oposición. El hecho de que $90.1 \%$ de los entrevistados afirmaron en su momento "haber visto siempre o a veces la franja" evidenció su nivel de penetración en las audiencias (Ortega, 1999). Su puesta en operación estuvo regulada por el artículo 31 de la Ley Orgánica Constitucional sobre Votaciones Populares y Escrutinios emitida en mayo de ese mismo año, y en la que se estipulaba que en el caso de plebiscito nacional los canales de televisión otorgarían 30 minutos diarios que se 
distribuirían la mitad para el gobierno y la otra mitad para los partidos "que sustenten posiciones diferentes a las del gobierno".

En Brasil, durante el proceso electoral de 1989, que marcaba el reestablecimiento de la elección directa para presidente de la república, el candidato Fernando Collor de Mello despliega su campaña privilegiando el espacio de los medios e introduce la publicidad negativa como recurso fundamental. En el Horario Gratuito de Propaganda Electoral advertía que de llegar Luís Inácio Lula da Silva, candidato del Partido del Trabajo, a la presidencia, confiscaría las cuentas de ahorros (Chaia, 2006).

En México, aun cuando en el proceso de 1988 aparecieron algunos elementos específicos de la mercadotecnia electoral-como la difusión de las encuestas electorales- es hasta 1994 que Ernesto Zedillo, candidato del entonces gobernante Partido Revolucionario Institucional, utiliza la publicidad política como un elemento sustantivo en su estrategia de campaña electoral; asimismo, aunque en forma limitada y con un enfoque de crítica al régimen priista, el Partido Acción Nacional aderezó su campaña con algunos spots publicitarios. Su irrupción se produjo, entonces, en el contexto de la reforma electoral de 1993 derivada de la crisis postelectoral de 1988, y en la cual se introdujo, por vez primera, "la reglamentación respecto a la contratación de tiempos comerciales en la radio y la televisión" (Aceves, 2000).

\section{LA “AMERICANIZACIÓN” DE LA POLÍTICA: \\ UN TÉRMINO A DEBATE}

Con la propagación del uso de los instrumentos mercadológicos en las contiendas electorales en diversidad de países, se ha convertido en un lugar común decir que la política se ha "americanizado". Ciertamente que el desarrollo de elementos propios del marketing político norteamericano, como la "personalización", el uso de encuestas, la participación de expertos en publicidad y creación de imagen en los equipos de campaña, y de manera especial el uso de los spots políticos, particularmente los televisivos, etcétera, han aparecido de manera cada vez mas frecuente en los países con procesos electorales que reivindican como democráticos. Ni siquiera los países europeos han logrado evadirse de esta "americanización”, como lo ilustra Castells (1999) con los ejemplos del Reino 
Unido y de España. En el caso del primero, aunque la publicidad pagada es ilegal, los partidos disponen de emisiones gratuitas tanto dentro como fuera de las campañas. En España, por su parte, el uso de los medios y la "personalización" cimentaron el arribo al poder de Felipe González en 1982. Incluso en Rusia, las campañas de estilo estadounidense hicieron su debut en las elecciones parlamentarias de 1993.

Empero, la diseminación del término "americanización" condujo a un debate entre los estudiosos de la comunicación política, respecto a la delimitación de los alcances del concepto. Por una parte, autores como Negrine y Papathanassopoulos (1996) advirtieron de los riesgos que podrían derivarse de interpretar esta adopción del "estilo" de campaña norteamericano, esta "americanización" como un proceso de "modernización" de la comunicación política en los países implicados. Es decir, que más allá de su empleo instrumental, el uso de las herramientas de la mercadotecnia política evidenciaba, en su desarrollo sociopolítico, un proceso de modernización del sistema político de la sociedad. Concretamente, se referían a la asimilación y reproducción de las características del sistema político americano.

Sin embargo, para el grueso de los autores que utilizan este término (Radunski 1996; Berlín Villafaña 2006; Zovatto, 2007) su significado se refiere estrictamente a la utilización de las herramientas de la mercadotecnia política -el uso de la encuestas y la publicidad política- que tuvieron su origen en Estados Unidos, pero con diversos grados de adopción y adaptación, se han convertido en el elemento central de las estrategias de comunicación de las campañas políticas en los procesos electorales en América Latina, como se desprende del comentario de Zovatto a propósito de las campañas electorales de 2006 en algunos países de este continente:

Por último, debe subrayarse el tema de las campañas electorales y su creciente "americanización". Un fenómeno en aumento, que se caracteriza por la personalización de la política, la preeminencia del candidato sobre el partido, el uso progresivo de la televisión y la descalificación del opositor por encima de las ideas en el marco de "campañas negativas". En esta línea se desarrollaron las campañas en Brasil, Costa Rica, Nicaragua, México y Perú (Zovatto, 2007). 


\section{EL PROCESO DEMOCRATIZADOR}

\section{Y LAS LEGISLACIONES ELECTORALES DE AMÉRICA LATINA}

No es casual que la irrupción de la mercadotecnia política y el diseño de campañas centradas en la comunicación mediática haya ocurrido en las dos últimas décadas del siglo pasado. Su aparición coincide con el desarrollo de procesos democratizadores en un conjunto de países latinoamericanos. Analistas del desarrollo político en América Latina como Zovatto y Orozco (2008), coinciden en términos generales con la "teoría de las olas" y ubican la emergencia democratizadora en Latinoamérica, en el periodo que Huntington (1994) denominó "la tercera ola", que abarcaba de 1974 a 1990. Por su parte, Peter Smith en su estudio sobre el desarrollo de la democracia en los países latinoamericanos afirma que su mayor crecimiento se observa en lo que denomina como "el tercer ciclo" de la democratización, que va de 1978 al 2000, cuando "casi el 90\% de la población de América Latina disfrutaba de la democracia electoral" (2004:196).

Ecuador retornó a la democracia en 1979, y en Chile, con la llegada a la presidencia de Patricio Aylwin en 1990, postulado por una coalición de partidos entre los que se encuentran la Democracia Cristiana y el Partido Socialista, se reestablece la democracia. Tal vez uno de los indicadores de este proceso democratizador lo constituye el desarrollo de las legislaciones electorales de los países latinoamericanos. De hecho, a excepción de Costa Rica y Brasil, cuyas legislaciones datan de 1952 y 1965 respectivamente, en los demás países sus legislaciones han sido elaboradas de la década de los ochenta en adelante.

Un caso peculiar es el de Colombia, que a raíz de la reforma constitucional mediante la cual se permitía la reelección del presidente, se elaboró una legislación especial, a la que se denominó Ley 996 Ley de Garantías Electorales, promulgada en noviembre de 2005, mediante la cual se definieron las reglas a que deberían ajustarse las elecciones presidenciales.

\section{PROCEDIMIENTO METODOLÓGICO}

El corpus de investigación se conformó con las legislaciones electorales que se encontraban vigentes en 2006, de 19 países latinoamericanos. 


\begin{tabular}{|c|c|c|}
\hline \multicolumn{3}{|c|}{$\begin{array}{l}\text { LEGISLACIONES ELECTORALES DE AMÉRICA LATINA } \\
\text {-VIGENTES AL 2006- }\end{array}$} \\
\hline País & Año & Legislación \\
\hline Argentina & 1983 & Código Electoral Nacional \\
\hline Bolivia & 1984 & Código Electoral \\
\hline Brasil & 1965 & Institui o Código Eleitoral \\
\hline Chile & $1988^{*}$ & $\begin{array}{l}\text { Ley Orgánica Constitucional sobre Votaciones } \\
\text { Electorales y Escrutinios } \\
\text { Ley } 19.884 / 2003 \text { sobre Transparencia, Límite } \\
\text { y Control del Gasto Electoral }\end{array}$ \\
\hline Colombia & 2005 & Ley 996 de 2005 Ley de Garantías Electorales \\
\hline Costa Rica & $1952 * *$ & Código Electoral. Ley No. 1536 \\
\hline Ecuador & 2000 & $\begin{array}{l}\text { Codificación de la Ley de Elecciones } \\
\text { Ley Orgánica de Control de Gasto Electoral } \\
\text { y de la Propaganda Electoral de } 2000\end{array}$ \\
\hline El Salvador & 1993 & Código Electoral \\
\hline Guatemala & 1985 & Ley Electoral y de Partidos Políticos \\
\hline Haití & 1999 & Loi Electorale \\
\hline Honduras & 2004 & Ley Electoral y de las Organizaciones Políticas \\
\hline México & $1990 * * *$ & $\begin{array}{l}\text { Código Federal de Instituciones y } \\
\text { Procedimientos Electorales }\end{array}$ \\
\hline Nicaragua & 2000 & Ley Electoral de Nicaragua \\
\hline Panamá & 2003 & Código Electoral \\
\hline Paraguay & 1996 & Código Electoral \\
\hline Perú & 1997 & $\begin{array}{l}\text { Ley Orgánica de Elecciones } \\
\text { Ley de Partidos Políticos de } 2003\end{array}$ \\
\hline R.Dominicana & 1997 & Ley Electoral No. 275-97 \\
\hline Uruguay & 1999 & Ley de Elecciones \\
\hline Venezuela & 1997 & $\begin{array}{l}\text { Ley Orgánica del Sufragio y Participación } \\
\text { Política }\end{array}$ \\
\hline
\end{tabular}

Fuente: elaboración propia

* Actualizada al 2003

** Actualizada al 2001

*** Actualizada al 2002 
Su selección se realizó sobre la base del cumplimiento de dos aspectos. El primero, que desde la perspectiva geopolítica fueran considerados como parte de América Latina, y segundo, que su regulación legislativa contemplara el desarrollo de campañas políticas en las contiendas electorales. ${ }^{2}$ Por otra parte, es necesario advertir que una característica de la regulación sobre las campañas electorales es que se encuentra diseminada en diversas legislaciones (Lauga y García Rodríguez, 2007). Comprenden desde el Texto Constitucional a Códigos y Leyes sobre Procesos Electorales, pasando por leyes sobre partidos políticos, hasta regulaciones específicas, como el financiamiento a los partidos así como algunas emanadas de la normatividad estatal y municipal. Sin embargo, para este análisis se incluyeron solamente aquellas legislaciones expresamente relacionadas con el proceso electoral.

Un primer dato que salta a la vista al realizar el análisis de las leyes electorales es su ausencia de homogeneidad. En efecto, tanto en el tipo como en la cantidad de aspectos relacionados con las campañas políticas, que son regulados por las legislaciones, se aprecia una notable diversidad. Cada país imprime en su normatividad aquellas cuestiones que consideran relevantes y prioritarias.

En su estudio sobre la regulación de las campañas políticas en las legislaciones latinoamericanas, Lauga y García Rodríguez (2007) distinguen cinco elementos que sirven de base para su análisis comparativo: a) las condiciones para su realización, b) su periodo y duración, c) las actividades tradicionales de proselitismo político, d) la campaña electoral por los medios de difusión, y e) prohibiciones y restricciones generales. Empero, sin dejar de reconocer que constituye un esfuerzo por agrupar los distintos aspectos sometidos a regulación en cinco categorías, su carácter harto general proporciona una visión muy limitada y poco específica sobre la manera en que las legislaciones electorales de los diversos países regulan las actividades de las campañas políticas, particularmente las relacionadas con la publicidad mediática. Por ello, para la elaboración de este trabajo se procedió a diseñar un instrumento

2 En el caso de Cuba, el artículo 171 de la Ley Electoral de 1992 señala textualmente que las actividades de los candidatos no son consideradas "campaña de propaganda electoral”. 
que hiciera posible la identificación de todos y cada uno de los aspectos relacionados con las actividades de campaña que se convertían en objeto de regulación.

Como primer paso se procedió a localizar en las legislaciones electorales los artículos relacionados con las campañas políticas. En seguida se confeccionó, con base en la técnica de análisis de contenido, una hoja de trabajo en la que se identificaron aquellos aspectos de la campaña política que eran sometidos a regulación. Este procedimiento puso al descubierto un elemento que se convirtió en el criterio básico para establecer una clasificación de los aspectos sometidos a regulación. Se identificó que el tratamiento que las legislaciones otorgaban a dichos aspectos presentaban notables diferencias tanto en el peso como en el alcance regulatorio. En el primer caso, la regulación se restringía a la definición del marco normativo para la realización de las actividades de campaña; en el segundo caso se prohibía expresamente la ejecución de determinadas actividades, y en el tercero se establecían sanciones mediante las cuales se castigaba al infractor. Esta triple cualidad en la regulación -normativa, prohibición y sanción- proporcionó el modelo básico en el que se sustentó el análisis comparativo, es decir, el primer nivel de comparación: qué aspectos regulan y cómo los regulan.

En atención, entonces, a sus características, se clasificó a los aspectos contemplados en la regulación de las legislaciones en tres tipos diferentes: 1) aspectos bajo norma, 2) aspectos prohibidos y 3) aspectos con sanción. En aras de mayor claridad habría que especificar que la tipología de "aspectos bajo norma" se refiere a los que la normatividad se concreta a la definición de los requisitos y/o los límites del acto o procedimiento realizado, por ejemplo, la definición de los límites del periodo de campaña. Por "aspectos prohibidos" se entiende aquellos sobre los que se emite tácitamente la prohibición al ejercicio de determinada actividad, por ejemplo, la difusión de propaganda electoral fuera del periodo permitido. Finalmente en la tipología "aspectos bajo sanción" se incluyen aquellos cuya ejecución es causa de una pena, sea pecuniaria o corporal, por ejemplo, la penalización de seis meses de cárcel por difundir propaganda fuera del periodo permitido.

Al concluir la revisión de las legislaciones se identificaron un total de 63 aspectos relacionados con las campañas electorales que se 
encontraban sujetos a regulación: 26 de ellos correspondían a la tipología de "aspectos bajo norma", 18 a la de "aspectos prohibidos" y 20 a la de "aspectos bajo sanción". Sin embargo estos aspectos, a pesar de compartir la característica que los agrupaba en determinada tipología, se referían a actividades o procedimientos que correspondían a una diversidad de factores que guardaban poca relación entre sí, lo que representaba una seria dificultad para llevar a cabo cualquier ejercicio de comparación. Por eso, atendiendo a sus "características específicas", se procedió a establecer un segundo nivel de clasificación, en el que se definieron seis categorías: 1) aspectos que regulan las "condiciones y requisitos" a cumplirse en la contienda electoral; 2) aspectos relacionados con el "acceso a los medios" de comunicación por parte de los contendientes; 3) aspectos relacionados con los contenidos de la "publicidad y la propaganda" electoral; 4) aspectos relacionados con la "cobertura informativa" que los medios masivos realizan de las campañas electorales (que solamente aparece en las categorías de aspectos bajo norma y bajo sanción); 5) aspectos relativos a los recursos y a las "actividades de gobierno"; y 6) aspectos "otros" que tienen que ver con diversas acciones o procedimientos de las campañas electorales.

En estas categorías se enmarcaron los 64 aspectos regulados por las legislaciones, y que para el propósito del presente trabajo se consideraron como "subcategorías" (ver cuadro 2).

\section{AMÉRICA LATINA Y CAMPAÑAS MEDIÁTICAS:}

\section{LOS CLAROSCUROS DE SU REGULACIÓN}

Esta metodología hizo posible identificar con un mayor grado de precisión los diversos aspectos relacionados con las campañas políticas que eran objeto de regulación en los respectivos países. Asimismo permitió identificar, mediante su recurrencia en las diferentes legislaciones, aquellos aspectos a los que se otorgaba mayor importancia. Pero, además, se pudo advertir con mucha claridad la ausencia de homogeneidad respecto al énfasis regulatorio de los diversos países. Es decir, que aunque los 64 aspectos sometidos a regulación sirvieron de base a las 197 menciones que se observaron en las legislaciones electorales, solamente un puñado de estos se hizo presente en un número significativo de 


\section{CUADRO 2}

TIPOS, CATEGORÍAS Y SUBCATEGORÍAS DE ANÁLISIS

\begin{tabular}{|c|c|c|}
\hline Tipo & Categorías & Subcategorías \\
\hline \multirow{6}{*}{ 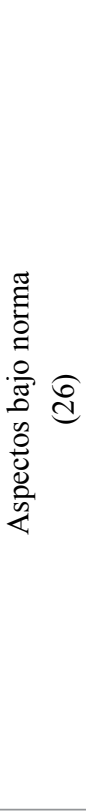 } & $\begin{array}{l}\text { Condiciones } \\
\text { y requisitos }\end{array}$ & $\begin{array}{l}\text { Definición de campaña, periodo de difusión, } \\
\text { equidad, derecho de uso de medios, libertad } \\
\text { de propaganda, obligatoriedad de transmisión, } \\
\text { censura o gravamen, responsabilidad, } \\
\text { identificación partido }\end{array}$ \\
\hline & $\begin{array}{l}\text { Publicidad y } \\
\text { propaganda }\end{array}$ & Definición de campaña y/o publicidad electoral \\
\hline & $\begin{array}{l}\text { Acceso a } \\
\text { medios }\end{array}$ & $\begin{array}{l}\text { Contratación, tarifas, espacios máximos, } \\
\text { catálogo tiempos horarios, información } \\
\text { contratación, tope gastos campaña, medios y } \\
\text { tiempo del Estado }\end{array}$ \\
\hline & $\begin{array}{l}\text { Cobertura } \\
\text { informativa }\end{array}$ & $\begin{array}{l}\text { Monitoreo de medios, equilibrio informativo, } \\
\text { derecho de réplica, uso de radio y TV }\end{array}$ \\
\hline & $\begin{array}{l}\text { Actividades } \\
\text { de gobierno }\end{array}$ & Propaganda estatal \\
\hline & Otros & $\begin{array}{l}\text { Uso espacios abiertos o cerrados, altoparlantes, } \\
\text { retiro de propaganda, comunicación en la jornada, } \\
\text { órgano de regulación }\end{array}$ \\
\hline
\end{tabular}

\begin{tabular}{|c|c|c|}
\hline \multirow{5}{*}{ 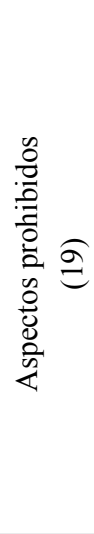 } & $\begin{array}{l}\text { Condiciones } \\
\text { y requisitos }\end{array}$ & Difusión fuera del periodo \\
\hline & $\begin{array}{l}\text { Publicidad } \\
\text { y propaganda }\end{array}$ & $\begin{array}{l}\text { Propaganda negra, propaganda anónima, } \\
\text { propaganda violenta, uso símbolos patrios, uso } \\
\text { símbolos religiosos, aludir otras naciones }\end{array}$ \\
\hline & $\begin{array}{l}\text { Acceso } \\
\text { a medios }\end{array}$ & $\begin{array}{l}\text { Tarifas, contratación por terceros, uso de medios, } \\
\text { contratar y dirigir programas periodísticos }\end{array}$ \\
\hline & $\begin{array}{l}\text { Actividades } \\
\text { de gobierno }\end{array}$ & $\begin{array}{l}\text { Transmisión presidencial en canal del Estado, } \\
\text { prohibiciones al presidente, servidores públicos, } \\
\text { recursos del Estado, publicidad estatal }\end{array}$ \\
\hline & Otros & $\begin{array}{l}\text { Uso espacio público, edificios públicos, } \\
\text { volumen alto }\end{array}$ \\
\hline
\end{tabular}




\begin{tabular}{|c|c|c|}
\hline Tipo & Categorías & Subcategorías \\
\hline \multirow{6}{*}{ 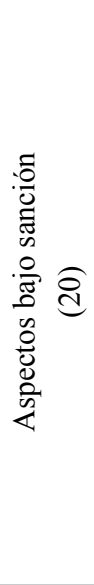 } & $\begin{array}{l}\text { Condiciones } \\
\text { y requisitos }\end{array}$ & $\begin{array}{l}\text { Difusión fuera periodos, incumplimiento en } \\
\text { general, identificación partido, responsabilidad }\end{array}$ \\
\hline & $\begin{array}{l}\text { Publicidad y } \\
\text { propaganda }\end{array}$ & $\begin{array}{l}\text { Propaganda negra, propaganda anónima, uso } \\
\text { símbolos patrios, espacios máximos, destrucción } \\
\text { de propaganda }\end{array}$ \\
\hline & $\begin{array}{l}\text { Acceso a } \\
\text { medios }\end{array}$ & $\begin{array}{l}\text { Contratación, remisión de tarifas, tope gastos } \\
\text { campaña }\end{array}$ \\
\hline & $\begin{array}{l}\text { Cobertura } \\
\text { informativa }\end{array}$ & Derecho de réplica \\
\hline & $\begin{array}{l}\text { Actividades } \\
\text { de gobierno }\end{array}$ & $\begin{array}{l}\text { Servidores públicos, uso medios públicos, } \\
\text { tiempo del Estado, publicidad estatal }\end{array}$ \\
\hline & Otros & $\begin{array}{l}\text { Regalos a electores, uso lengua extranjera, } \\
\text { participación extranjeros }\end{array}$ \\
\hline
\end{tabular}

Fuente: elaboración propia.

países. Fue precisamente este dato de la mención sobre determinados aspectos en las legislaciones lo que constituyó el elemento clave para efectos del estudio comparativo.

De entrada, este acercamiento puso en evidencia algunos rasgos que caracterizan a las legislaciones latinoamericanas sobre la materia. En primer lugar se advierte que el énfasis legislativo se concentra en emitir preceptos que solamente "regulan" diversas cuestiones relacionadas con las campañas políticas. Es decir que se limitan a ofrecer definiciones, normas, criterios, límites, etcétera, a dichas actividades; de hecho el total de menciones sobre aspectos "bajo norma" (103) representa $52.3 \%$ del total. En contraste, la cantidad de menciones sobre aspectos "prohibidos" (56) representa solamente $28.4 \%$ y con un porcentaje considerablemente menor, sólo $19.3 \%$ del total de menciones (38), se encuentran aquellos aspectos "bajo sanción" a los que la legislación establece una pena por su ejecución.

Esto significa que las legislaciones electorales en Latinoamérica tienen un carácter fundamentalmente normativo, es decir, que sus regulaciones se redactan en términos del "deber ser" y no un carácter disciplinario en el que se establezcan las bases del "así tiene que ser". 
Se trata, pues, de legislaciones que apelan a la voluntad de los actores y no establecen las medidas correctivas que desalienten o castiguen la falta de observancia de la normatividad establecida, es decir, de legislaciones insuficientes, limitadas, "sin dientes", cuyos preceptos quedan a nivel de "llamadas a misa".

En segundo lugar, si se atiende la distribución de menciones con relación a las diversas categorías de los aspectos, se observa que el rubro que obtuvo el mayor número de menciones fue el de "condiciones y requisitos" con 60 , seguido por las categorías de "acceso a medios" con 42 y "publicidad y propaganda" con 41 . Se advierte, entonces, que si bien aquellos aspectos relacionados con las condiciones que deben privar para el desarrollo del proceso aparecen con mayor frecuencia en las legislaciones, son los rubros vinculados con el carácter mediático de las campañas -"acceso a medios" y "publicidad y propaganda" - los que considerados en conjunto representan $42.1 \%$ del total de menciones, y si le agregamos el relativo a "cobertura informativa" el porcentaje alcanza 45.6\%. Es decir, que en las legislaciones latinoamericanas el asunto de la dimensión mediática de las campañas políticas representa un elemento de primer orden. Resulta obvio que el reconocimiento del importante papel de los medios en las contiendas electorales se ha convertido en una preocupación para los legisladores. De ahí se explica el espacio dedicado en la normatividad, así como un mayor énfasis disciplinario que se observa en los aspectos relativos a los contenidos de la publicidad y propaganda. A diferencia de los otros rubros, en este el peso regulatorio tiene que ver con los "aspectos prohibidos" y las "sanciones".

\section{LA REGULACIÓN DE LAS CAMPAÑAS \\ EN LA LEGISLACIÓN ELECTORAL}

Arriba se mencionaba que una característica de las legislaciones latinoamericanas consistía en la ausencia de homogeneidad. Ni la cantidad ni el tipo de aspectos que son sometidos a regulación posibilitan establecer un patrón común que asimile a los diversos códigos y leyes. Sin embargo, es posible establecer una clasificación de los países conforme a la cantidad de aspectos regulados. Desde esta perspectiva, se pueden 


\section{CUADRO 3}

DISTRIBUCIÓN DE LOS ASPECTOS REGULADOS

EN LAS LEGISLACIONES ELECTORALES

NÚMERO DE MENCIONES

\begin{tabular}{lcccc} 
& $\begin{array}{c}\text { Aspectos } \\
\text { bajo norma }\end{array}$ & $\begin{array}{c}\text { Aspectos } \\
\text { prohibidos }\end{array}$ & $\begin{array}{c}\text { Aspectos } \\
\text { bajo sanción }\end{array}$ & Suma \\
\hline $\begin{array}{c}\text { Condiciones } \\
\text { y requisitos }\end{array}$ & 38 & 12 & 10 & 60 \\
$\begin{array}{c}\text { Publicidad } \\
\text { y propaganda }\end{array}$ & 11 & 21 & 10 & 42 \\
$\begin{array}{c}\text { Acceso a medios } \\
\text { Cobertura } \\
\text { informativa }\end{array}$ & 31 & 6 & 4 & 41 \\
Actividades & 6 & - & 1 & 7 \\
$\quad$ de gobierno & 4 & 10 & 10 & 24 \\
$\begin{array}{l}\text { Otros } \\
\text { Suma }\end{array}$ & 13 & 7 & 3 & 23 \\
\hline
\end{tabular}

Fuente: elaboración propia.

identificar cuatro bloques de países cuyo rango está determinado por el número de aspectos que contemplan en su legislación. Así, los países cuya legislación regula entre 1 y 5 aspectos quedarían incluidos entre los de "mínima regulación"; los que regulen de 6 a 10 aspectos, serían de "baja regulación"; los de 11 a 15 aspectos de "media regulación" y finalmente aquellos que contemplan 16 o más aspectos formarían el bloque de "alta regulación".

\section{ANÁLISIS COMPARADO}

DE LAS LEGISLACIONES LATINOAMERICANAS

Para el análisis comparativo entre las legislaciones de los diversos países se procederá a observar los aspectos conforme a las categorías establecidas -aspectos bajo norma, aspectos prohibidos y aspectos bajo sanción-, y dentro de cada una de ellas ubicar dichos aspectos en su clasificación correspondiente. 


\section{CUADRO 4}

CLASIFICACIÓN DE LOS PAÍSES CONFORME AL RANGO DE REGULACIÓN

\begin{tabular}{ll}
\multicolumn{1}{c}{ Rango } & \multicolumn{1}{c}{ Países } \\
\hline $\begin{array}{l}\text { Alta regulación } \\
\text { (más de } 15 \text { aspectos) }\end{array}$ & Colombia (22), México (18), Bolivia (17), \\
Media regulación & Brasil (14), Haití (14), Panamá (12) \\
$(11-15$ aspectos) & y Venezuela (11) \\
Baja regulación & Paraguay (9), Costa Rica (8), Argentina (7), \\
$(6-10$ aspectos) & El Salvador (7), Chile (6), Ecuador (6) y \\
& Nicaragua (6) \\
Mínima regulación & Perú (4), República Dominicana (3) y Uruguay (1) \\
$(1-5$ aspectos) & \\
\hline
\end{tabular}

Fuente: elaboración propia.

\section{ASPECTOS BAJO NORMA}

De las ocho subcategorías que conforman el rubro de "condiciones y requisitos", solamente dos de ellas se encuentran incorporadas en el cuerpo de la legislación de más de la mitad de los países analizados: "período de difusión" en 11 y "equidad" también en 11. Muy por debajo "responsabilidad sobre la propaganda" aparece en siete. En el rubro de "publicidad y propaganda", que en este caso la regulación se limitaba a la definición de los conceptos de campaña y/o propaganda, solamente 11 de los 18 países la incluyeron en su legislación. Por su parte, en el rubro "acceso a medios", que comprende a siete subcategorías, solamente el aspecto "tarifas" es incluido en nueve países; otros aspectos que alcanzan una cierta presencia son los de "contratación" con seis, además de "espacios máximos" y "medios y tiempo del Estado" en cinco cada uno. En contraste, el rubro de "cobertura informativa", solamente el aspecto relacionado con el "monitoreo de medios", aparece en tres legislaciones. El rubro de "actividades de gobierno", que solamente contempla la subcategoría de "propaganda estatal", aparece en cuatro. Finalmente en el rubro "otros", los aspectos que tienen alguna presencia en la normatividad son los relacionados 
con el "órgano de regulación" que se encuentra en seis y el de "uso de espacios abiertos o cerrados" que aparece en tres.

Así, encontramos que en relación a los 26 aspectos cuya regulación se circunscribe a la definición "normativa" de las condiciones y los límites a que deben apegarse los actores políticos, solamente en tres de ellos - periodo de difusión (11), equidad (11) y tarifas (9)- han sido regulados por más de la mitad de las legislaciones. La regulación sobre la "responsabilidad sobre propaganda" se aprecia en siete; “contratación" de publicidad política en seis; los "espacios máximos" y los "medios y tiempos del Estado" se encuentran en cinco; la regulación sobre la "propaganda estatal" aparece en cuatro; los demás aspectos aparecen a lo sumo en tres legislaciones y ocho de ellos solamente se encuentran en una.

Sin embargo, los resultados del cuadro 5 ilustran de alguna manera aquellos puntos que a juicio de los legisladores requieren una regulación específica. Destaca que luego de la medida de establecer con precisión los periodos de duración de las campañas, la normatividad se enfoque hacia un aspecto que resulta central para garantizar el desarrollo de una contienda apegada a principios democráticos: la equidad. Pero un elemento que ligado al anterior merece una especial consideración es el relativo a la regulación sobre las tarifas que las empresas de medios imponen a la publicidad política. El hecho de que nueve países la incluyan en su legislación ilustra dos cosas: por una parte, la penetración de la mercadotecnia política en las estrategias de comunicación de los actores políticos y, por otra, la importancia fundamental de la publicidad mediática en las contiendas electorales.

Pero el cuadro también ilustra las prioridades de las diversas legislaciones sobre los aspectos a regular. No se advierte homogeneidad en esta selección, incluso en los países que se encuentran en el grupo de “alta regulación". Así, aunque al aspecto relativo a la delimitación del periodo lo incorporan en sus respectivas legislaciones, el aspecto de "equidad" solamente lo incluyen dos (Colombia y Bolivia). En contraste, dicho aspecto se encuentra establecido en la mayoría de países considerados de "baja regulación".

Asimismo, los aspectos relacionados con el uso de la publicidad política -contratación, tarifas, espacio máximo y tope de gastos de cam- 


\section{CUADRO 5}

ASPECTOS “BAJO NORMA” EN LAS LEGISLACIONES ELECTORALES

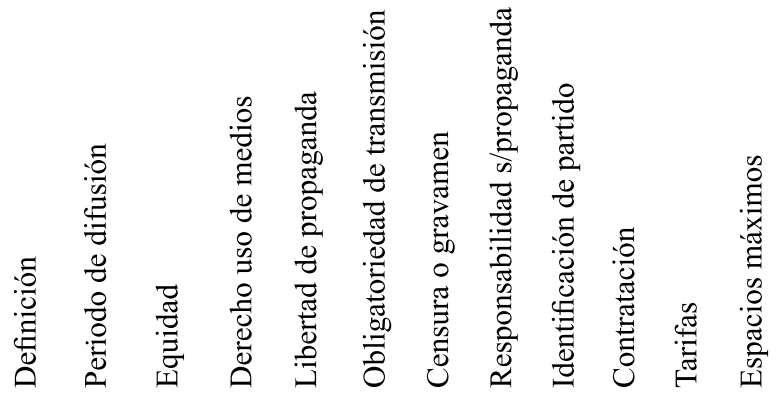

Colombia $\mathrm{x} \quad \mathrm{x}$

\begin{tabular}{llllllllll} 
México & $\mathrm{x}$ & $\mathrm{x}$ & & & & $\mathrm{x}$ & $\mathrm{x}$ & $\mathrm{x}$ & \\
\hline Bolivia & $\mathrm{x}$ & $\mathrm{x}$ & $\mathrm{x}$ & $\mathrm{x}$ & $\mathrm{x}$ & $\mathrm{x}$ & $\mathrm{x}$ & $\mathrm{x}$ \\
\hline Guatemala & $\mathrm{x}$ & $\mathrm{x}$ & & & & & $\mathrm{x}$ & $\mathrm{x}$ & $\mathrm{x}$
\end{tabular}

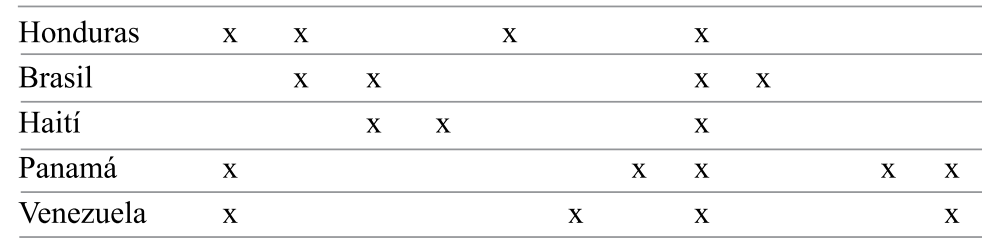

\begin{tabular}{lcccccccc}
\hline Paraguay & $\mathrm{x}$ & $\mathrm{x}$ & $\mathrm{x}$ & & & & $\mathrm{x}$ \\
\hline Costa Rica & & & $\mathrm{x}$ & & $\mathrm{x}$ & $\mathrm{x}$ & $\mathrm{x}$ \\
\hline Argentina & $\mathrm{x}$ & $\mathrm{x}$ & & & & & \\
\hline El Salvador & & $\mathrm{x}$ & $\mathrm{x}$ & & & $\mathrm{x}$ & \\
\hline Chile & $\mathrm{x}$ & $\mathrm{x}$ & & & & & & \\
\hline Ecuador & $\mathrm{x}$ & $\mathrm{x}$ & $\mathrm{x}$ & $\mathrm{x}$ & & & & \\
\hline Nicaragua & & & $\mathrm{x}$ & & $\mathrm{x}$ & $\mathrm{x}$ & $\mathrm{x}$ \\
\hline Perú & & $\mathrm{x}$ & & & & & \\
\hline R. Dominicana & & $\mathrm{x}$ & & & & &
\end{tabular}

\section{Uruguay}

\begin{tabular}{lllllllllllll}
\hline SUMA & 11 & 11 & 11 & 3 & 1 & 1 & 2 & 7 & 2 & 6 & 9 & 5
\end{tabular}

Fuente: elaboración propia. 


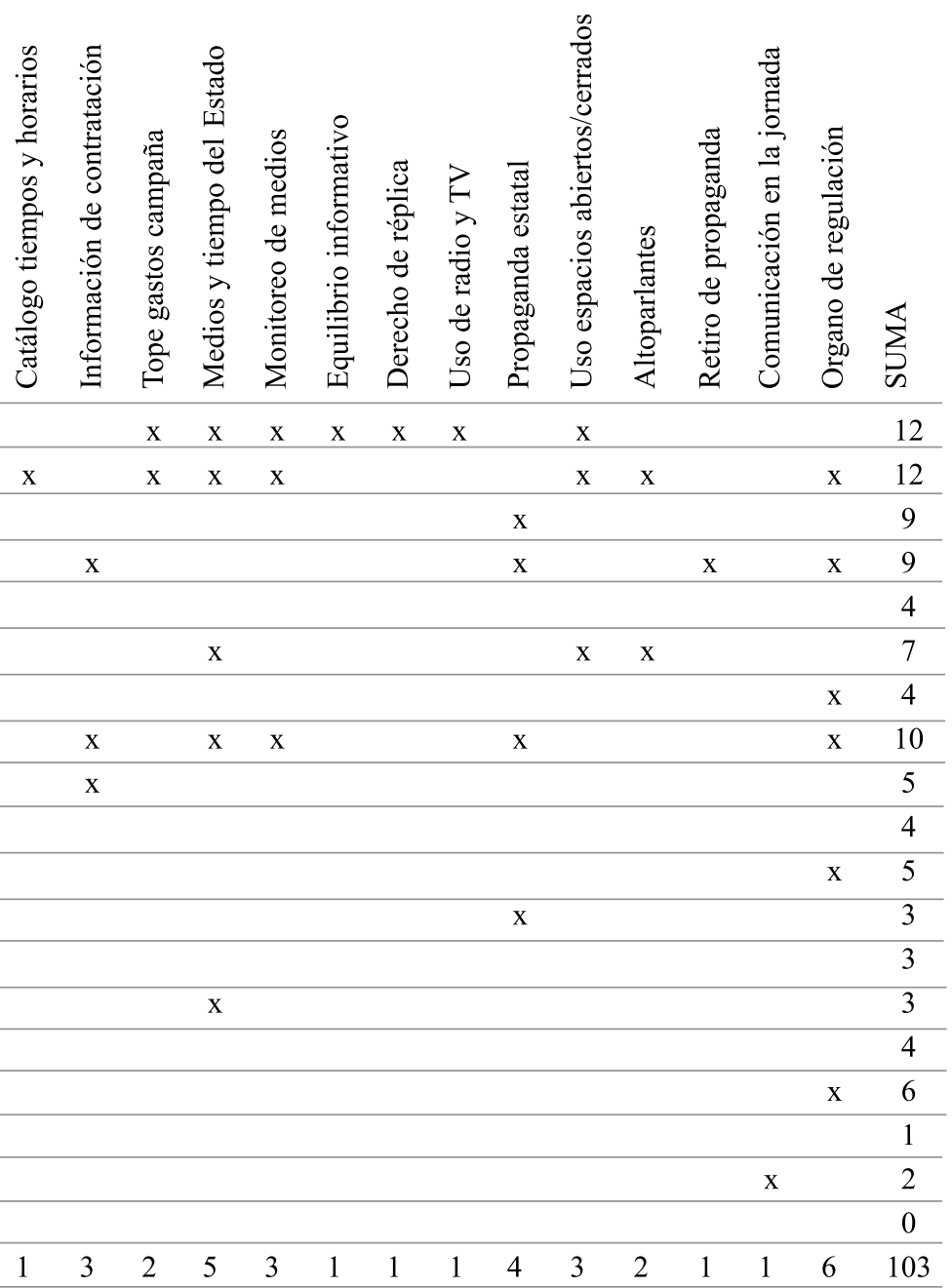


paña- reciben un tratamiento preferencial en las legislaciones de "alta regulación", a excepción de Honduras. En ello se suman países como Panamá, Paraguay, Costa Rica, El Salvador y Nicaragua.

Un caso especial lo reviste el hecho de que solamente 11 de los 19 países incorporan en el texto de su normativa alguna definición sobre lo que se debe entender por campaña electoral o, en su defecto, de propaganda electoral. En el primer caso, que comprende a los países de Argentina, Bolivia, Colombia, Ecuador, Honduras, México y Venezuela, coinciden en definir a la campaña electoral como el conjunto de actividades que los candidatos y partidos realizan con el propósito expreso de captar el sufragio, a la que las legislaciones de Bolivia, Ecuador y Honduras agregan la difusión de los programas de gobierno. En todos estos casos, ya en forma implícita o explícita se incluye a la propaganda electoral como parte integrante de la campaña, y en el caso particular de Colombia se especifica el carácter presidencial.

Por su parte, mientras que algunos de los países que incluyen una definición sobre propaganda electoral -Panamá, Paraguay y Méxicocircunscriben su alcance a la difusión de mensajes por cualquier medio, incluyendo los electrónicos, para la difusión de las candidaturas y el llamado al voto, otros - Chile y Honduras- enfatizan la función persuasiva de la propaganda en el sentido de la inducción del voto por determinados candidatos. En contraste, Guatemala incluye en el concepto de propaganda todas aquellas actividades proselitistas que las otras legislaciones incluyen en su definición de "campaña".

A diferencia de los demás países, Brasil, Costa Rica, El Salvador, Nicaragua, Perú, República Dominicana y Uruguay, no consideraron incluir entre los aspectos regulados por su legislación una definición de campaña electoral o de propaganda política.

\section{ASPECTOS PROHIBIDOS}

En el caso de las actividades explícitamente prohibidas en las legislaciones electorales, el rubro de "publicidad y propaganda" es el que registra mayor número de referencias (21) seguido por los rubros de "condiciones y requisitos" (12) y "actividades de gobierno" (10). Pero además, a excepción del rubro "cobertura informativa" al que no se 
menciona en ninguna de ellas, los rubros de "acceso a medios" (6) y "otros" (7) se encuentran presentes en la tercera parte de las mismas.

Sin duda que los aspectos que reciben una prohibición expresa en la normatividad electoral, aparte de la difusión de las campañas fuera de los tiempos establecidos, se relacionan directamente con los contenidos de la propaganda política. Son 13 los países que la incluyen en su legislación, sea en la acepción de "propaganda negra", de "propaganda anónima" o de "propaganda violenta". En contraste, dicha prohibición se encuentra ausente en las regulaciones de Argentina, Colombia, Chile, Nicaragua, Panamá y Perú. El otro rubro que obtiene mayor presencia entre los aspectos prohibidos es el relacionado con las actividades relacionadas con las labores de gobierno (10). Entre ellas, destaca de nuevo la relacionada con el uso irregular de la publicidad estatal (4), que expresamente se encuentra prohibida por Argentina, Colombia, Guatemala y Perú.

El énfasis otorgado a la prohibición de actividades relacionadas con la propaganda, específicamente la denominada propaganda negra o publicidad negativa, expresa la valoración que los legisladores le otorgan como un factor que influye en la definición del voto, así como el deseo de desterrar sus efectos perniciosos sobre la contienda política de las arenas electorales.

\section{ASPECTOS BAJO SANCIÓN}

Líneas arriba se señalaba que las legislaciones electorales se enfocaban básicamente solamente a regular, en el sentido de establecer el "deber ser", las actividades relacionadas con las campañas políticas y que existía una diferencia sustancial entre los aspectos solamente regulados y aquellos por cuya ejecución se establece una sanción. Los 20 aspectos regulados solamente son mencionados en 38 ocasiones.

$\mathrm{El}$ análisis de las legislaciones indica que el único aspecto a sancionar en el que coinciden siete países, es el relativo a la "difusión fuera de periodo", que corresponde al rubro de "condiciones y requisitos". Otros cinco países coinciden en sancionar algunos de los aspectos relacionados con la propaganda política. Las sanciones a las actividades irregulares de los servidores o el uso indebido de los recursos públicos se establecen en 


\section{CUADRO 6}

ASPECTOS “PROHIBIDOS” EN LAS LEGISLACIONES ELECTORALES

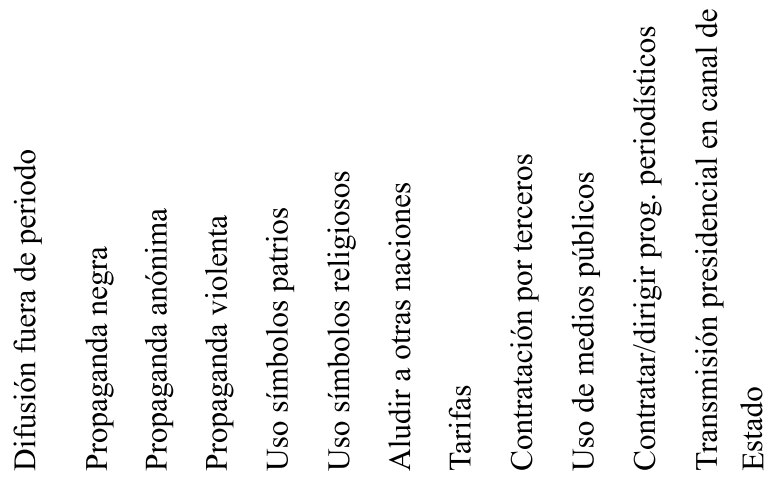

\begin{tabular}{|c|c|c|c|c|c|c|c|c|c|c|c|c|}
\hline Colombia & $\mathrm{x}$ & & & & $\mathrm{x}$ & & & & & & $\mathrm{x}$ & $\mathrm{x}$ \\
\hline México & & $\mathrm{x}$ & & & & $\mathrm{x}$ & & & $\mathrm{x}$ & & & \\
\hline Bolivia & $\mathrm{x}$ & $\mathrm{x}$ & $\mathrm{x}$ & & & & & & & & $\mathrm{x}$ & \\
\hline Guatemala & $\mathrm{x}$ & & $\mathrm{x}$ & & & & & $\mathrm{x}$ & & & & \\
\hline Honduras & $\mathrm{x}$ & & $\mathrm{x}$ & & $\mathrm{x}$ & $\mathrm{x}$ & & & & $\mathrm{x}$ & & \\
\hline Brasil & $\mathrm{x}$ & & $\mathrm{x}$ & & & & & & & & & \\
\hline Haití & $\mathrm{x}$ & & & $\mathrm{x}$ & & & & & & & & \\
\hline \multicolumn{13}{|l|}{ Panamá } \\
\hline Venezuela & & $\mathrm{x}$ & $\mathrm{x}$ & & $\mathrm{x}$ & & & & & & & \\
\hline Paraguay & & $\mathrm{x}$ & & & & & $\mathrm{x}$ & & & & & \\
\hline Costa Rica & $\mathrm{x}$ & $\mathrm{x}$ & & & & & & & & & & \\
\hline Argentina & $\mathrm{x}$ & & & & & & & & & & & \\
\hline El Salvador & $\mathrm{x}$ & $\mathrm{x}$ & & & & & & & & & & \\
\hline Chile & $\mathrm{x}$ & & & & & & & $\mathrm{x}$ & & & & \\
\hline Ecuador & $\mathrm{x}$ & & $\mathrm{x}$ & & & & & & & & & \\
\hline \multicolumn{13}{|l|}{ Nicaragua } \\
\hline Perú & $\mathrm{x}$ & & & & & & & & & & & \\
\hline \multicolumn{13}{|c|}{ R. Dominicana } \\
\hline \multicolumn{13}{|c|}{ Uruguay } \\
\hline SUMA & 12 & 8 & 6 & 1 & 3 & 2 & 1 & 2 & 1 & 1 & 2 & 1 \\
\hline
\end{tabular}

Fuente: elaboración propia. 


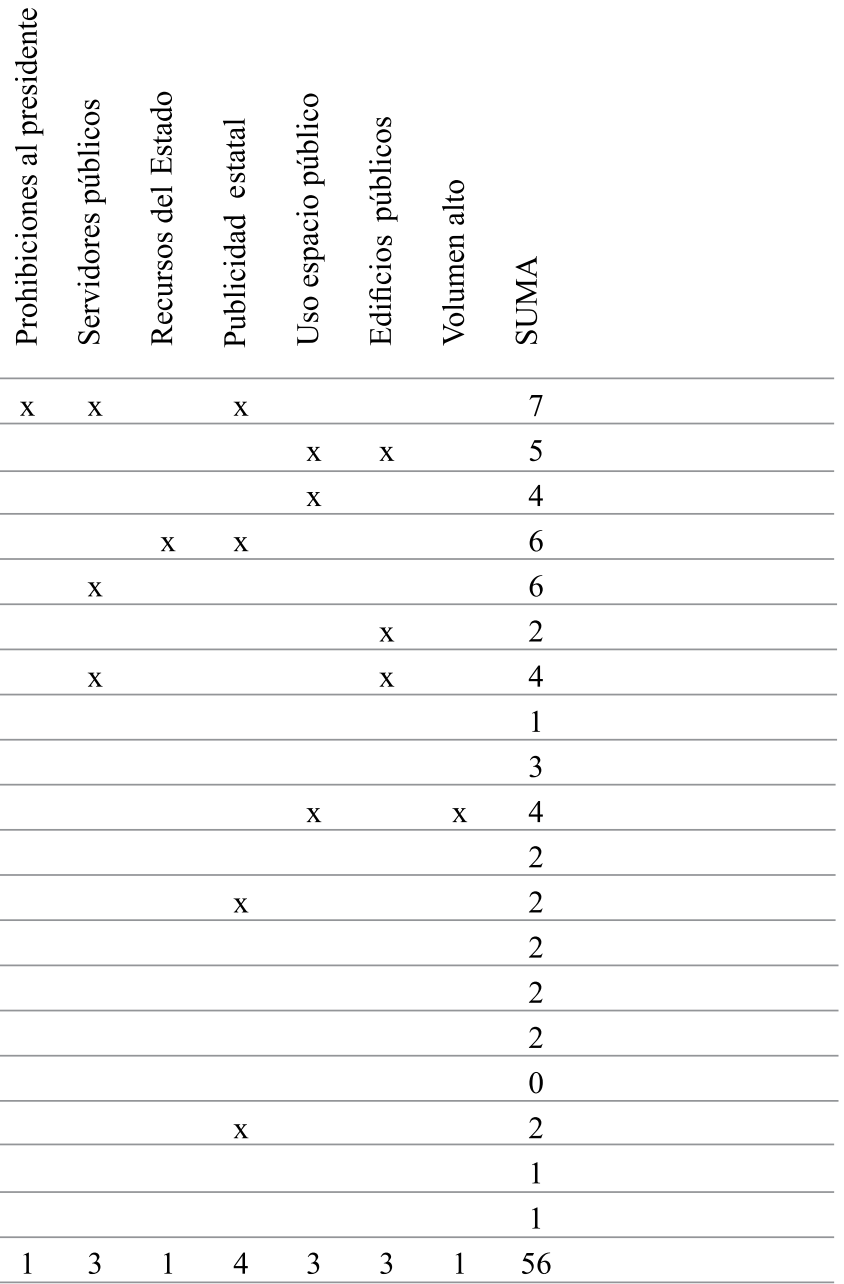


siete países, y solamente en cuatro, todos ellos ubicados entre los países de "alta regulación", crean sanciones relacionadas a la difusión de la publicidad mediática, referida a la "contratación, remisión de tarifas y topes de campaña".

\section{CUADRO 7}

ASPECTOS “BAJO SANCIÓN” EN LAS LEGISLACIONES ELECTORALES

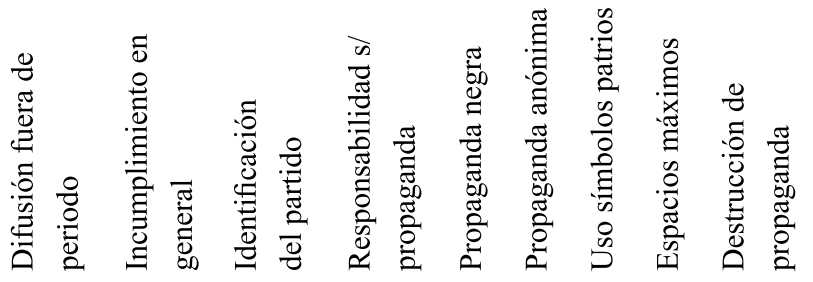

Colombia

México

Bolivia

$\mathrm{X}$

$\mathrm{X}$

Guatemala

\begin{tabular}{|c|c|c|c|c|}
\hline Honduras & $\mathrm{x}$ & $\mathrm{x}$ & $\mathrm{x}$ & $\mathrm{x}$ \\
\hline Brasil & & & & $\mathrm{x}$ \\
\hline Haití & $\mathrm{x}$ & & & $\mathrm{x}$ \\
\hline
\end{tabular}

Panamá $\quad \mathrm{x}$

Venezuela $\mathrm{x} \quad \mathrm{x} \quad \mathrm{x}$

Paraguay $\quad \mathrm{x}$

Costa Rica $\quad \mathrm{x}$

Argentina $\quad \mathrm{X}$

El Salvador $\mathrm{x}$

Chile $\mathrm{x}$

Ecuador

Nicaragua

Perú

R. Dominicana

Uruguay

$\begin{array}{llllllllll}\text { SUMA } & 7 & 1 & 1 & 1 & 3 & 2 & 1 & 1 & 3\end{array}$

Fuente: elaboración propia. 


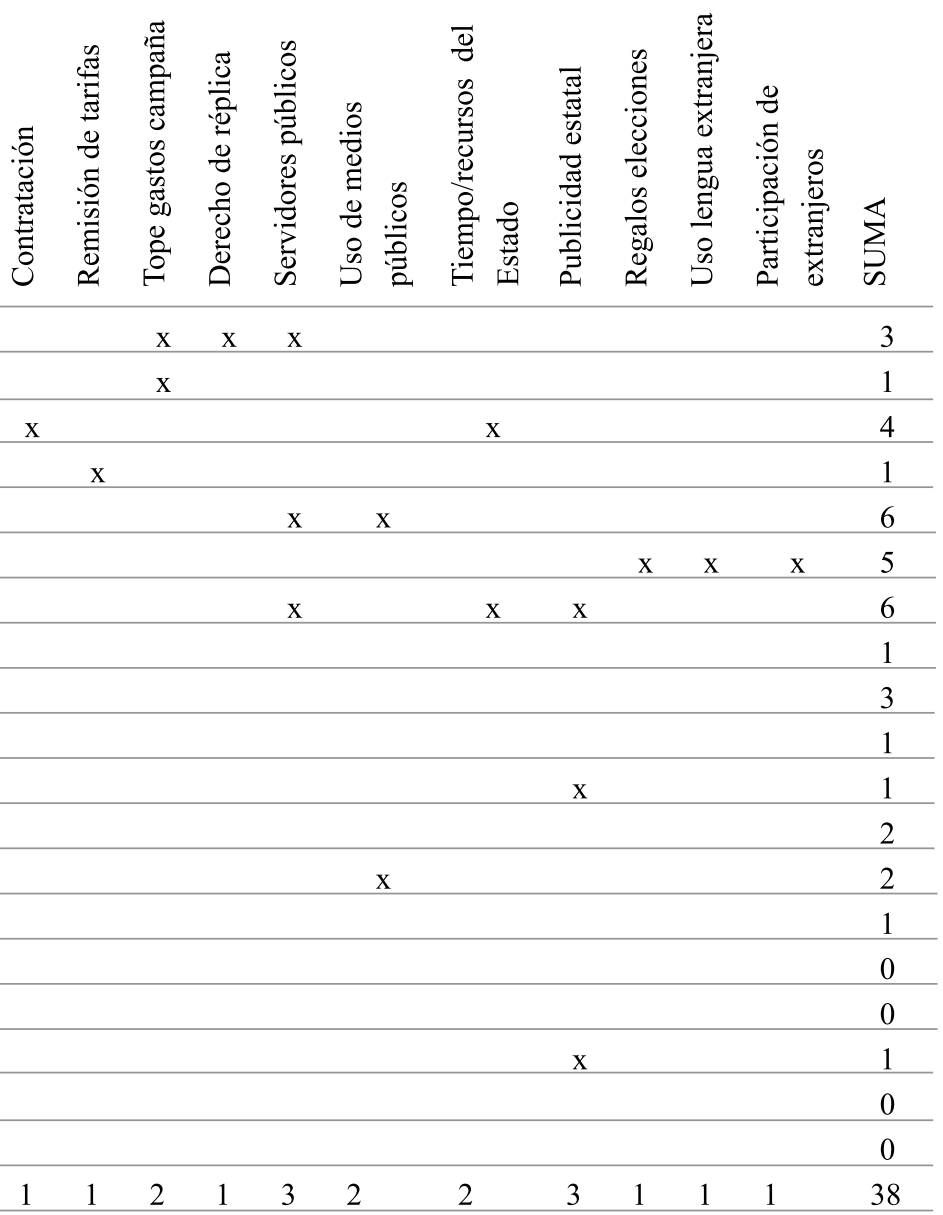




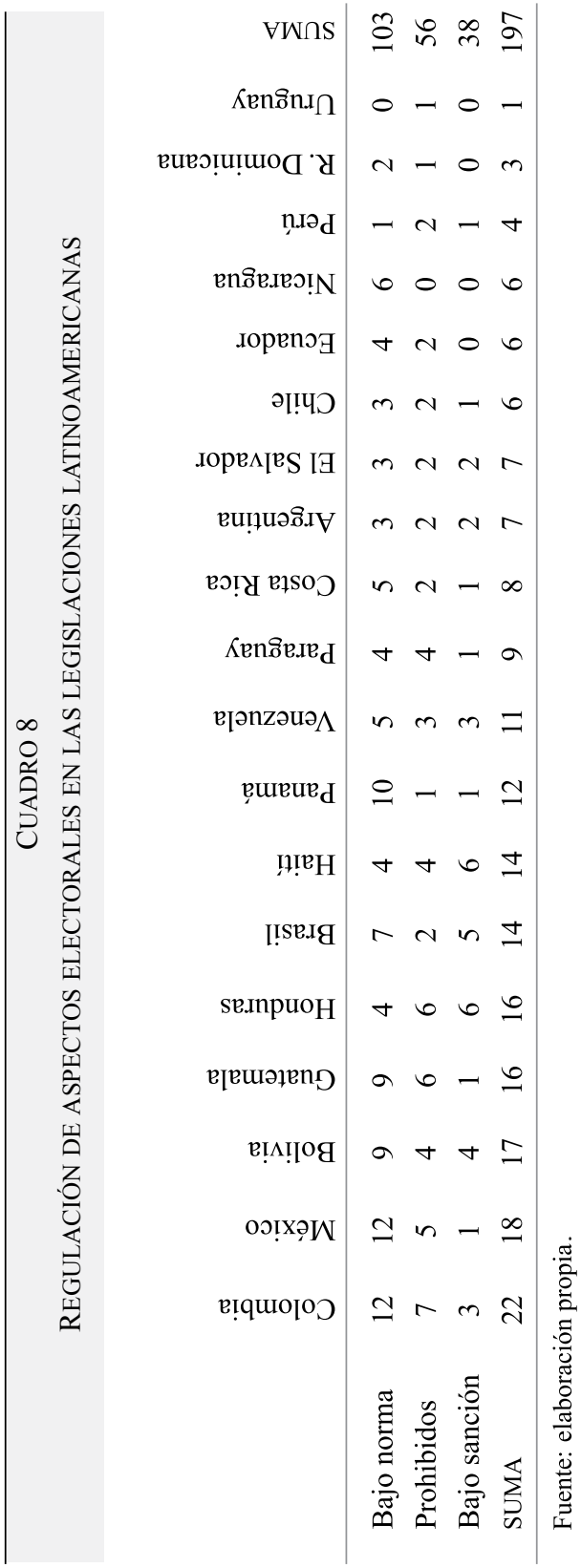


Si se considera que la efectividad de la observancia de las leyes se garantiza por la capacidad de sancionar las conductas y actividades que se desvían de la norma, se podría concluir que las legislaciones electorales de los países latinoamericanos presentan un nivel muy bajo de efectividad. Pero inclusive dentro de esta consideración se aprecian diferencias importantes. En un extremo estarían países como Honduras y Haití, que establecen sanciones a seis aspectos, seguidos por Brasil y Bolivia que sancionan a cinco y cuatro, respectivamente; en el otro, Ecuador, Nicaragua, República Dominicana y Uruguay, que no establecen sanción alguna.

Por otra parte, se puede apreciar que no existe relación entre las legislaciones de países considerados de "alta regulación", con el número de aspectos sancionados. El ejemplo más palpable lo suministran México y Guatemala, que a pesar de que en sus regulaciones abarcan 18 y 16 aspectos respectivamente, solamente a uno se le establece una sanción. En contraste, Argentina y El Salvador, que pertenecen al grupo de "baja regulación", sancionan a dos.

\section{CONCLUSIONES PROVISIONALES}

El análisis de la regulación de las campañas electorales y la publicidad política en las legislaciones de 19 países de América Latina permite adelantar algunas conclusiones, así sean preliminares.

La primera tiene que ver con las diferencias sustanciales que se observan entre las legislaciones, tanto en el número de aspectos sometidos a regulación como a la calidad de los mismos. Esta disparidad parece ser el resultado de las particularidades con las que cada país ha experimentado sus procesos electorales. Así, la estabilidad de la norma permanece en tanto resulte funcional para procesar los conflictos derivados de la contienda electoral; en caso contrario se generan procesos de reforma.

La segunda se refiere al profundo rezago que se observa en la legislación electoral respecto a la centralidad del espacio mediático, que se ha convertido en la principal arena de confrontación y debate político. Inclusive en aquellos países catalogados como de "alta regulación" en el presente escrito, la normatividad establecida resulta insuficiente. 
La tercera conclusión es que el énfasis regulatorio se restringe a establecer la norma de los aspectos relacionados con las actividades de campaña y publicidad política en el ámbito del "deber ser", sin establecer paralelamente a los ordenamientos la aplicación de sanciones correspondientes a los infractores. Son, pues, como se dice en el argot jurídico, legislaciones "sin dientes".

Pero además de las anteriores, se impone reflexionar acerca de si la promulgación de leyes en la materia garantiza la calidad democrática de los procesos electorales, vale decir, si la legislación abona a la democratización del sistema político. Esta pregunta, que no admite una respuesta categórica, remite de nuevo a la particularidad de la experiencia de cada uno de los países. Se podría decir que la efectividad de la norma depende de dos cuestiones: de la manera en que los actores políticos y sociales observan la norma establecida, y de la manera en que la norma adquiere el peso suficiente para obligar a su observancia.

\section{Bibliografía}

ACEVES González, F. de J. (2000). La investigación académica sobre el papel de los medios de comunicación en los procesos electorales en México. Comunicación y Sociedad, núm. 37. Guadalajara: Universidad de Guadalajara, enero-junio.

BARRERA, A. (2008). Ecuador: desafíos y oportunidades del proceso electoral 2006. Nueva Sociedad, edición especial, marzo de 2006. Consultado 11 de abril, http://www.nuso.org/docesp/barrera_final.pdf.

BERLÍN Villafaña, I. (2006). México: Campañas electorales americanizadas. Chasqui, núm. 96, CIESPAL, diciembre.

CASTELLS, M. (1999). La era de la información, vol. II. México: Siglo XXI.

CHAIA, V. (2006). "Los medios y las elecciones en Brasil", ponencia presentada en el I Foro de Comunicación, Educación y Ciudadanía, 27 de marzo, Pamplona, España. Traducción al español de Martín Aranguri.

DAHLGREN, P. (1997). El espacio público y los medios. ¿Una nueva era?. En Isabelle Veyrat-Masson y Daniel Dayan (comps.), Espacios públicos en imágenes. España: Gedisa. 
DomínguEZ, J. (1983). Cómo vender a un candidato. El producto político en el mercado electoral venezolano. Nueva Sociedad, núm. 68 septiembre-octubre, 147.

HUNTINGTON, S. (1994). La tercera ola. La democratización a finales del siglo $X X$. España: Paidós.

IYENGAR, S. y Kinder, D. R. (1993). Televisión y opinión pública. Información es poder. México: Ed. Gernika.

LAUGA, M. y García Rodríguez, J. I. (2007). La campaña electoral: publicidad/propaganda, período, prohibiciones. En Nohlen, Zovatto, Orozco, Thompson (comps.), Tratado de derecho electoral comparado de América Latina. México: FCE.

MAAREK, P. J. (1997). Vote, marketing político y comunicación. Claves para una buena información politica. España: Paidós Comunicación.

MCCOMBS, M. and Kiousis, S. (2004). Agenda-Setting effects and attitude strength political figures during the 1996 presidential election. Communication Research, vol. 31, núm. 1, February. Sage Publications.

MuRARO, H. (1990). La publicidad política (y la política de la publicidad) en la Argentina. Diálogos de la Comunicación, núm. 27, julio. Perú: FELAFACS.

NEGRINE, R. and Papathanassopoulos, S. (1996). The "Americanization" of Political Comunication. A critique. Harvard International Journal of Press/Politics.

ORTEGA, F. (1999). Una aproximación de la elección parlamentaria Chile 1997. En Priess y Tuesta Soldevilla (eds.), Campañas electorales y medios de comunicación en America Latina (p. 166). Argentina: CIEDLA/KAS.

RADUNSKI, P. (1999). Management de la comunicación política. La americanización de las campañas electorales. En Priess, F. y Thesing, J. (eds.), Globalización, democracia y medios de comunicación. Buenos Aires: CIEDLA.

SARTORI, G. (1992). Elementos de teoría política. (cap. 15). Madrid: Alianza Universidad Textos.

- (1998). Homo videns. La sociedad teledirigida. México: Taurus. 
SHAH, D. V., Domke, D. and Wackman, D. B. (1996). To Thine Owb Self Be True: Values, Framing, and Voter Decision-Making Strategies. Communication Research, vol. 23, núm. 5, October. Sage Publications.

SMith, P. (2004). Los ciclos de la democracia electoral en América Latina. 1900-2000. Política y gobierno, vol . XI, núm. 2, II semestre.

SteVenson, R. Rainer Böhme, L. Nickel, N. (2001). The TV Agenda-Setting influence on campaign 2000. Egyptian Journal of Public Opinion Research, 2.

ZOVATTO, D. (2007). América Latina después del "rally" electoral 2005-2006: algunas tendencias y datos sobresalientes. Nueva Sociedad, núm. 207, enero-febrero.

- y Orozco H., J. (2008). Reforma política y electoral en América Latina 1978-2007: lectura regional comparada. En Zovatto y Orozco (coords.), Reforma política y electoral en América Latina 19782007. México: UNAM/IDEA. 